\title{
The microRNA-15a-PAl-2 axis in cholangiocarcinoma-associated fibroblasts promotes migration of cancer cells
}

\author{
Penkhae Utaijaratrasmi ${ }^{1}$, Kulthida Vaeteewoottacharn ${ }^{2,5}$, Takaaki Tsunematsu ${ }^{6}$, Pranisa Jamjantra ${ }^{9}$, \\ Sopit Wongkham ${ }^{2,5}$, Chawalit Pairojkul ${ }^{3}$, Narong Khuntikeo ${ }^{4,5}$, Naozumi Ishimaru' ${ }^{6}$, Yongyut Sirivatanauksorn? \\ Ananya Pongpaibul ${ }^{8}$, Peti Thuwajit ${ }^{9}$, Chanitra Thuwajit ${ }^{9^{*}}$ (D) and Yasusei Kudo ${ }^{6^{*}}$
}

\begin{abstract}
Background: Cholangiocarcinoma (CCA) has an abundance of tumor stroma which plays an important role in cancer progression via tumor-promoting signals. This study aims to explore the microRNA (miRNA) profile of CCA-associated fibroblasts (CCFs) and the roles of any identified miRNAs in CCA progression.

Methods: miRNA expression profiles of CCFs and normal skin fibroblasts were compared by microarray. Identified downregulated miRNAs and their target genes were confirmed by real-time PCR. Their binding was confirmed by a luciferase reporter assay. The effects of conditioned-media (CM) of miRNA mimic- and antagonist-transfected CCFs were tested in CCA migration in wound healing assays. Finally, the levels of miRNA and their target genes were examined by real-time PCR and immunohistochemistry in clinical CCA samples.

Results: miR-15a was identified as a downregulated miRNA in CCFs. Moreover, PAI-2 was identified as a novel target gene of miR-15a. Recombinant PAI-2 promoted migration of CCA cells. Moreover, CM from miR-15a mimic-transfected CCFs suppressed migration of CCA cells. Lower expression of miR-15a and higher expression of PAI-2 were observed in human CCA samples compared with normal liver tissues. Importantly, PAI-2 expression correlated with poor prognosis in CCA patients.
\end{abstract}

Conclusions: These findings highlight the miR-15a/PAl-2 axis as a potential therapeutic target in CCA patients.

Keywords: Cancer-associated fibroblasts, microRNA (miRNA), Cholangiocarcinoma, PAl-2, Migration,

Tumor microenvironment

\section{Background}

Cholangiocarcinoma (CCA) is a tumor of bile duct epithelium which arises from cholangiocytes or the epithelial cells lining the biliary tree [1]. It is a major health problem in the endemic area of Opisthorchis viverrini infection in Southeast Asia especially in the northeastern part of Thailand [2]. It is well known that CCA predominantly shows an abundant desmoplastic reaction in the tumor stroma [3]. These alpha-smooth muscle actin (ASMA) positive stromal cells were correlated with the

\footnotetext{
* Correspondence: chanitra.thu@mahidol.ac.th; yasusei@tokushima-u.ac.jp ${ }^{9}$ Department of Immunology, Faculty of Medicine Siriraj Hospital, Mahidol University, Bangkok, Thailand

${ }^{6}$ Department of Oral Molecular Pathology, Tokushima University Graduate School of Biomedical Sciences, Tokushima, Japan

Full list of author information is available at the end of the article
}

degree of tumor fibrosis in CCA tissues [4] and levels were significantly related to poor prognosis of CCA patients [5].

Cancer-associated fibroblasts (CAFs) are the major component in the stromal environment of several carcinomas including CCA and mainly affect tumor progression through the induction of cancer cell growth, invasion, and metastasis $[6,7]$. The current authors have previously reported that CAFs in CCA tissues have a high expression of ASMA and the level of ASMApositive CAFs was correlated with poor patient survival [5]. In addition, the conditioned-media (CM) from CAFs induced CCA cell proliferation by driving cancer cells into the G2/M phase of the cycle. Previous exploration of the gene expression profile of CAFs isolated from CCA tissues found a set of genes which encoded 
secreted tumorigenic protein products that could act in a paracrine manner to promote cancer progression [8].

MicroRNAs (miRNAs) are small, non-coding singlestranded RNAs and one of the key post-transcriptional gene expression regulators $[9,10]$. They act as negative regulators to control gene expression via imperfect or perfect binding to their target mRNAs. miRNA regulation in CAFs has been studied in several cancers [11-17]. Microarray analyses identified specific expression of several miRNAs in CAF within urinary bladder cancer and breast cancer compared with fibroblasts from noncancer tissues $[12,16]$. The impact of miRNAs underlying gene expression control in CCA-associated fibroblasts (CCFs) has not so far been reported. This study aimed to explore the miRNA profiles of CCFs by miRNA microarrays and to identify the aberrant target mRNAs; in particular, those that encode secreted tumorigenic proteins.

\section{Methods}

\section{Cell culture}

Human CCA cell lines, KKU-213 and KKU-055 were cultured in Hams F-12 Nutrient Mixture (Invitrogen) supplemented with $10 \%$ heat-inactivated fetal bovine serum (FBS). Antibiotics including $0.1 \mathrm{U} / \mathrm{ml}$ penicillin $\mathrm{G}$ sodium and $0.1 \mathrm{mg} / \mathrm{ml}$ streptomycin (Gibco BRL), and $5 \mathrm{mg} / \mathrm{ml}$ amphotericin B (Amphotret ${ }^{\mathrm{m}}$, Bharat Serums and Vaccines Limited Ltd.) were added to prevent microbial contaminations. CCFs were isolated from CCA tissues obtained from patients who underwent hepatectomy at Srinakarind Hospital. The protocol was approved by the Khon Kaen University Ethics Committee for Human Research (HE521209 and HE571283). Each patient was informed about tissue collection and signed the consent form. The pieces of tissue were submerged in antibioticcontaining Dulbecco's Modified Eagle Media (DMEM; Gibco BRL) with 10\% FBS for 30-60 min to reduce microbial contamination during the process of sample collection and transfer. The samples were rinsed with $1 \mathrm{X}$ phosphate buffer saline (PBS) and cut into small pieces $\left(2 \mathrm{~mm}^{3}\right)$. Fresh complete medium, 10\% FBS-containing DMEM with $20 \mathrm{mM}$ HEPES (Gibco BRL), was used to wash out cell debris. Pieces of tissue were transferred to fresh culture vessels and allowed to adhere at $37^{\circ} \mathrm{C}$ in a $5 \% \mathrm{CO}_{2}$ in air incubator. After fibroblast-like cells emerged from beneath the tissue pieces, the adherent cells were trypsinized with $0.25 \%$ trypsin/EDTA (Gibco BRL). The complete medium was replaced every 3 days. Cells were subcultured for 7 to 23 passages and used for the reported studies. Normal skin fibroblasts (SFs) were obtained from human tissues and cultured in a similar way.

\section{Immunocytochemistry}

To identify primary cultures of fibroblasts from contaminating cancer cells, the absence of epithelial markers and the presence of fibroblast-related mesenchymal markers were checked. Cytokeratin 19 (CK19), an epithelial marker; vimentin (VIM) and alpha-smooth muscle actin (ASMA), mesenchymal markers, were selected. CCA cell lines were used as the positive controls for CK19 detection. Cells were cultured for $24 \mathrm{~h}$ in 96well plates and fixed with $4 \%$ paraformaldehyde for $15 \mathrm{~min}$ and permeabilized with $1 \%$ Triton X-100 for 1 min. Non-specific binding was blocked by $1 \%$ BSA in 1X PBS for ASMA and 5\% FBS in 1X PBS for VIM and CK19. The mouse anti-human CK19 antibody (1:100 dilution; SC-6278, Santa Cruz Biotechnologies Inc.), mouse anti-human VIM antibody (1:500 dilution; SC6260, Santa Cruz Biotechnologies Inc.), and mouse antihuman ASMA antibody (1:200 dilution; Sigma-Aldrich) were used. The primary antibody was incubated for $3 \mathrm{~h}$ at room temperature. After washing, goat anti-mouse IgG-Cy3 (Jackson Immunoresearch Laboratories Inc.) was added for $1 \mathrm{~h}$ at room temperature followed by adding Hoechst stain (Invitrogen). The signals were detected under an inverted fluorescence microscope.

\section{Fibroblast conditioned-media collection}

CM from CCFs and SFs was collected by growing cells in complete medium for 4 days until cells reached about 90\% confluency. Then, cells were washed twice with PBS and incubated in DMEM with 2\% FBS for $48 \mathrm{~h}$. After that, $\mathrm{CM}$ was collected, centrifuged at $4{ }^{\circ} \mathrm{C} 1000 \mathrm{~g}$ for $5 \mathrm{~min}$ to remove cell debris, sterile filtered and stored at $-80{ }^{\circ} \mathrm{C}$ until use.

\section{Wound healing migration assay}

CCA cells were cultured in 6-well plates until they reached approximately $90 \%$ confluence. A reference line was drawn in the center of the underside of the plate. The cell monolayer was scratched using a sterile $1000-\mu \mathrm{l}$ pipette tip followed by washing three times with fibroblast CM, serum-free medium or complete medium. The scratched areas indicated by reference lines were recorded at the beginning and $24 \mathrm{~h}$ after treatment. The migration efficiency was expressed as the percentage closure of the wound area calculated by the following formula:

$\%$ wound healing $=\frac{\text { wound space at } 0 \mathrm{~h} \text {-wound space at } 24 \mathrm{~h}}{\text { wound space at } 0 \mathrm{~h}} \times 100$

\section{Cell proliferation assay}

Cells were suspended with 5000 cells/well in 96-well plates for $24 \mathrm{~h}$. Culture medium was then changed to $100 \mu \mathrm{l} /$ well of $10 \% \mathrm{FBS}$ in DEMEM or CM and the plate was incubated for $24 \mathrm{~h}$ and $48 \mathrm{~h}$ in a $5 \% \mathrm{CO}_{2}$ incubator at $37^{\circ} \mathrm{C}$. Cell viability was measured by MTS assay (Promega, Mandison, WI) following manufacturer's instruction. The 
absorbance was detected at $490 \mathrm{~nm}$ with a microplate reader. The assays were performed in duplicate.

For checking the effect of recombinant target proteins, 8000 cells/well were suspended for $24 \mathrm{~h}$. Then, culture medium was changed to fresh medium with or without $2 \mu \mathrm{g} / \mathrm{ml}$ of human recombinant WNT10B protein or $2 \mu \mathrm{g} / \mathrm{ml}$ of human recombinant PAI-2 protein in 96-well plates and the plates were incubated for $24 \mathrm{~h}$. Finally, proliferating cell numbers were determined using the cell-counting kit 8 (CCK-8) solution (Dojindo Molecular Technique, Inc.). $10 \mu \mathrm{l}$ of CCK-8 solution was added to each well and incubated for $1 \mathrm{~h}$ in a $5 \% \mathrm{CO}_{2}$ incubator at $37{ }^{\circ} \mathrm{C}$. The number of proliferating cells was determined by absorbance measured at $450 \mathrm{~nm}$ using a microplate reader at 0,24 and $48 \mathrm{~h}$.

\section{miRNA microarray analysis}

miRNA expression profiles of CCFs and SFs were analyzed by the TaqMan ${ }^{\circ}$ Array Human MicroRNA Card A or Card B Set v2.0 (Applied Biosystems). Total RNA was extracted by MirVana ${ }^{\circ}$ microRNA isolation kit (Applied Biosystems). Then $480 \mathrm{ng}$ of total RNA was converted to cDNA by Megaplex ${ }^{\text {Tn }}$ RT stem-loop primer pools A and Megaplex ${ }^{\text {TI }}$ RT stem-loop primer pools B (Applied Biosystems) following the instruction manual. RT reaction was performed using Megaplex ${ }^{\mathrm{Tm}}$ RT primers, human pools A, B and TaqMan ${ }^{\circ}$ MicroRNA Reverse Transcription Kit (Applied Biosystems). cDNAs obtained were amplified with TaqMan ${ }^{\circ}$ Human MicroRNA Array A, B and TaqMan ${ }^{\circ}$ Universal Master Mix II, no UNG using Real time PCR ABI7900HT (Applied Biosystems). The amplification reactions were monitored using SDS software v2.3, and data analyzed with RQ Manger 1.2. The fold changes of each CCF sample were compared with average expression of SFs after normalization with U6 small nuclear RNA (snRNA). Fold changes $>2$-fold were taken to indicate up-regulated miRNAs and $\leq 0.5$ fold down-regulated miRNAs.

\section{Real-time PCR}

To evaluate miRNAs, total RNA was isolated from cells using the MirVana ${ }^{\circ}$ microRNA isolation kit (Applied Biosystems). The isolates were quantified and their purity was evaluated using a spectrophotometer. The cDNA was synthesized from $10 \mathrm{ng}$ of total RNA using a TaqMan ${ }^{\circ}$ MicroRNA Assay and a TaqMan ${ }^{\circ}$ MicroRNA Reverse Transcription Kit (Applied Biosystems). Synthesized cDNA was amplified with a TaqMan ${ }^{\circ}$ MicroRNA Assay and a TaqMan ${ }^{\circ}$ Universal Master Mix II, no UNG (Applied Biosystems) using an ABI 7300 real time PCR system (Applied Biosystems) for 45 cycles of denaturation at $95{ }^{\circ} \mathrm{C}$ for $15 \mathrm{~s}$, and annealing and extension at $60{ }^{\circ} \mathrm{C}$ for $60 \mathrm{~s}$. Data were normalized with U6 and each analysis was performed three times. The average of three trials was used for statistical analysis. ANOVA was used for comparison of variables in more than two groups and a Student's T-test was used for comparisons between two groups. A $P$-value $<0.05$ was considered statistically significant.

To evaluate mRNAs, $1 \mu \mathrm{g}$ of total RNA was converted to cDNA by ReverTra Ace- $\alpha^{\circ}$ (Toyobo Co; Ltd) and $50 \mathrm{ng}$ of cDNA was amplified with $\mathrm{SYBR}^{\circ}$ Premix Ex Taq $^{\text {Tw }}$ II (Takara Bio Inc.). The reaction was performed using a DNA Engine Opticon Real-Time Thermal Cycler (MJ Research, Inc.) under the following conditions; heatdenaturation at $95{ }^{\circ} \mathrm{C}$ for $10 \mathrm{~min}$ followed by cycled amplification at $95^{\circ} \mathrm{C}$ for $15 \mathrm{~s}$ and $60^{\circ} \mathrm{C}$ for $45 \mathrm{~s}$ for $50 \mathrm{cy}-$ cles. The primer sequences are shown in Additional file 1: Table S1 and Additional file 2: Table S2. Data were normalized with GAPDH and each analysis was performed three times. The average of three trials was used for statistical analysis. The ANOVA test was used for comparison of variables in more than two groups and the Students T-test was used for two groups. A $P$-value $<0.05$ was considered statistically significant.

\section{miRNA target gene prediction}

The potential target genes of miR-15a and miR-148a were determined using TargetScanHuman 6.2 (http:// targetscan.org/). To further reduce the false target genes in the analysis, target genes that were copredicted target genes by at least two of the three data sets were considered as potential targets. According to these algorithms, protein array chip data and mRNA microarray chip data from a previous report [8] were used for screening the most suitable candidate target genes. Candidate targeted genes which encoded the secreted proteins, were selected as targeted genes from overlapping at least 2 of 3 algorithms or TargetScan and have been found in a previous report [8] and/or previous reports from PubMed data.

\section{Transfection of miRNA mimic and miRNA inhibitors}

The hsa-miRNAs mimics were commercially available (Ambion ${ }^{\circ}$, Thermo Scientific). The product numbers MH10235 and MH1026 were used as a miR-15a and miR148a mimics. The negative control miRNA mimic (mirVana ${ }^{\text {tx }}$ miRNA mimic negative control \#1) was used as the scrambled miRNA mimic. The anti-mir-15a and miR-148a inhibitors, MC10235 and MC10263, were obtained from Ambion, Thermo Scientific, whereas a negative control inhibitor, mirVana ${ }^{\text {Tw }}$ miRNA inhibitor negative control \#1, were used as the scrambled miRNA inhibitor. Cells (CCFs) were transfected with $5 \mathrm{nM}$ hsamiR-15a, hsa-miR-148a and used as the negative control miRNA mimic using Lipofectamine RNAi Max (Invitrogen) according to the manufacturer's protocol. Cells (SFs) were transfected with $75 \mathrm{nM}$ of miR-15a inhibitor, 
miR-148a inhibitor and negative control miRNA inhibitor using Lipofectamine RNAi Max. Efficiency of miRNA inhibition or mimic transfection was confirmed at the mRNA level by real-time PCR.

\section{Luciferase reporter assay}

The full-length 3'-UTR of human VEGFA, PAI-2, and WNT10B was amplified by PCR from genomic DNA and cloned at the EcoRI site into pmirGLO vector (Promega). For PAI-2, we also generated a deletion mutant of putative miR-15a binding sequences, TGCTGCT, from full-length 3 '-UTR of human PAI-2 construct. We used a deletion mutant of pmirGLOPAI-2 as a negative control. This construct was confirmed by sequencing. For luciferase activity analysis, this vector was co-transfected with miRNA duplex in 293 T cells using DharmaFECT Duo transfection reagent (Thermo Fisher Scientific) for $72 \mathrm{~h}$, and luciferase assays were performed with the Dual-Luciferase reporter system (Promega) according to the manufacturer's instructions. Luminescent signal was quantified by a luminometer (Glomax; Promega), and each value from the firefly luciferase construct was normalized against the Renilla luciferase assay.

\section{Double chamber migration assay}

24-well cell culture inserts with $8 \mu \mathrm{M}$ pores (3097, Falcon, Becton Dickinson) were used. After trypsinization, $1.5 \times 10^{5} \mathrm{CCA}$ cells were resuspended in $\mathrm{CM}$ under different conditions and placed in the upper compartments of the cell culture inserts for $7 \mathrm{~h}$. In an additional assay, $2 \mu \mathrm{g} / \mathrm{ml}$ of human recombinant WNT10B protein (7196-WN-010, R\&D systems) or $2 \mu \mathrm{g} / \mathrm{ml}$ of human recombinant PAI-2 protein (P6129, Abnova) was added after trypsinization and before plating the cells on the upper compartments of the inserts. After incubation at $37{ }^{\circ} \mathrm{C}$, the cells that migrated through the membrane to the lower side were fixed with formalin and then stained by hematoxylin. The number of migrated cells were determined by counting the cells on the lower side of the filter under a microscope at $\times 100$ magnification. The filters were assayed 3 times and 3 fields were randomly selected and counted for each assay.

\section{Western blot analysis}

Sample protein concentrations were measured by the Coomassie Plus ${ }^{\mathrm{Tm}}$ (Bradford) Assay Kit (Thermo scientific), and $30 \mu \mathrm{g}$ total protein/lane were subjected to electrophoresis on $10 \%$ polyacrylamide gels followed by electroblotting onto polyvinylidene fluoride (PVDF) membranes. The membranes were blocked with $3 \%$ milk in TBS-T and incubated overnight at $4{ }^{\circ} \mathrm{C}$ with antihuman PAI-2 polyclonal antibody (Santa Cruz). The membranes were then washed with TBS-T and incubated with HRP-conjugated goat anti-rabbit IgG antibody (ab6721, Abcam, Cambridge, MA, USA) and the proteins visualized using SuperSignal West Pico Chemiluminescent Substrate under Gel Document Synene (Syngene). $\beta$-actin was used as a loading control. For CM, PAI-2 level was quantitated by densitometric analysis of the protein bands against that of the total protein loading using ImageJ software (National Institutes of Health, Bethesda, MD, USA).

\section{Immunohistochemistry in CCA tissues}

Seventy-four cases of intrahepatic CCA were used in this study. All patients were preoperatively chemo- and radiotherapy naïve. All tissue samples with their clinicohistopathological records were obtained from the Department of Pathology, Faculty of Medicine Siriraj Hospital, Mahidol University Faculty of Medicine and Departments of Biochemistry, Pathology, and Surgery Faculty of Medicine, Khon Kaen University under the approval of the Ethics Committee for Human Research (HE521209 and HE571283) and Siriraj Institutional Review Board (si287/2011). The demographic data of all the patients in this study are summarized (Table 1).

Tissue sections were deparaffinized in xylene, rehydrated in descending grades of ethanol. Heat-induced antigen retrieval was performed in a $95{ }^{\circ} \mathrm{C}$ water bath for $1 \mathrm{~h}$ by incubation with $0.05 \mathrm{M}$ EDTA buffer $\mathrm{pH}$ 8.0. Endogenous peroxidase activity was blocked with methanol containing $0.3 \% \mathrm{H}_{2} \mathrm{O}_{2}$ for 30 min followed by blocking non-specific binding sites with $3 \%$ bovine serum albumin in $1 \times$ PBS for $1 \mathrm{~h}$ at room temperature. The sections were treated with polyclonal human PAI-2 antibody (Santa Cruz, 1:100) at $4{ }^{\circ} \mathrm{C}$ overnight. The EnVision $^{+}$System-HRP labelled polymer anti-rabbit (K4003, Dako, Carpinteria, CA, USA) secondary antibody was used. The immunoreactive signal was developed by Liquid $\mathrm{DAB}^{+}$Substrate Chromogen System (K3467, Dako) and counterstained with hematoxylin. Tissue slides were dehydrated, mounted, and further analyzed using a ScanScope ${ }^{\circ}$ scanner. Sections were semi-quantitatively evaluated and scored by two pathologists; both were blinded to the clinical parameters. For grading the immunohistochemical results, the staining intensities were recorded separately for cancer cells and CCFs. The I scale of intensity: 0 (negative), 1 (weak), 2 (moderate), and 3 (strong) was used whereas the percentage of positive cells $(\mathrm{P})$ in the area was scored as: 0 , 1 (1-25\%), 2 (26-50\%), 3 (51-75\%) and 4 (>75\%). The multiplication of $\mathrm{P}$ and I resulted in the total score of 0-12. The PAI-2 immunohistochemical score of more than 6 was classified as the high expression group, whereas those with $\leq 6$ total score was graded as the low expression group. 
Table 1 Univariate analysis and Cox regression multivariate analysis of PAI-2 in cancer cells in clinical CCA samples and patient clinicopathological parameters

\begin{tabular}{|c|c|c|c|c|c|c|}
\hline \multirow[t]{3}{*}{ Parameters (no. of cases) } & & \multicolumn{3}{|c|}{ Univariate } & \multicolumn{2}{|l|}{ Multivariate } \\
\hline & & \multicolumn{2}{|c|}{ PAl-2 in cancer cells } & \multirow[t]{2}{*}{$P$-value } & \multirow{2}{*}{$\begin{array}{l}\text { OR } \\
(95 \% \mathrm{Cl})\end{array}$} & \multirow[t]{2}{*}{$P$-value } \\
\hline & & High & Low & & & \\
\hline High PAl-2 expression (72) & & 45 & 27 & - & $3.452(1.188-10.030)$ & 0.023 \\
\hline \multirow[t]{2}{*}{ Age (y) (72) } & $<60$ & 24 & 13 & 0.808 & $1.00(0.499-2.003)$ & 1.000 \\
\hline & $\geq 60$ & 21 & 14 & & & \\
\hline \multirow[t]{2}{*}{ Gender (72) } & Male & 25 & 17 & 0.625 & $1.291(0.683-2.443)$ & 0.432 \\
\hline & Female & 20 & 10 & & & \\
\hline \multirow[t]{2}{*}{ Tumor size (cm) (67) } & $<5$ & 15 & 9 & 0.790 & $1.375(0.698-2.705)$ & 0.357 \\
\hline & $\geq 5$ & 29 & 14 & & & \\
\hline \multirow[t]{2}{*}{ Tumor staging (72) } & I - III & 8 & 10 & 0.093 & $0.963(0.359-2.579)$ & 0.940 \\
\hline & IV & 37 & 17 & & & \\
\hline \multirow[t]{4}{*}{ Histological type (72) } & WD & 23 & 9 & 0.220 & - & 0.008 \\
\hline & MD & 6 & 4 & 1.000 & $2.034(0.915-4.519)$ & 0.081 \\
\hline & PD & 2 & 5 & 0.095 & 4.414 (1.1610-12.106) & 0.004 \\
\hline & Pap & 14 & 9 & 1.000 & 6.199 (1.754-21.909) & 0.005 \\
\hline \multirow[t]{2}{*}{ Vascular invasion (51) } & Absence & 18 & 16 & 0.065 & $0.405(0.190-0.866)$ & 0.020 \\
\hline & Presence & 14 & 3 & & & \\
\hline \multirow[t]{2}{*}{ LN metastasis (72) } & Absence & 28 & 20 & 0.439 & $2.078(0.925-4.670)$ & 0.077 \\
\hline & Presence & 17 & 7 & & & \\
\hline
\end{tabular}

WD well differentiated, $M D$ moderately differentiated, $P D$ poorly differentiated, Pap papillary, OR odd ratio, $95 \%$ CI $95 \%$ confidence interval, $L N$ lymph node

\section{Statistical analyses}

The differences between the test and the control conditions in this study were statistically analyzed from the mean \pm SD of three independent experiments. Chisquare or Fisher's exact tests were used to analyze the clinicopathohistological relevance of PAI-2 in clinical CCA samples. The Kaplan-Meier method and Log-Rank tests were used for survival analysis. Univariate and multivariate Cox regression analyses of potential factors were performed to identify the significant predictors. All the statistical analyses were performed using SPSS 17.0 statistical software. $P$-values of less than 0.05 were considered to be statistically significant.

\section{Results}

\section{Characterization of CCFs isolated from CCA tissues}

The CCFs (B149 and C096) and normal control SFs (SFA3 and SFA5) showed spindle-like shapes in contrast to the cobble-stoned shape of KKU-213 CCA cells (Fig. 1a). In CCFs and SFs, immunocytochemical analysis revealed negative staining of the epithelial marker CK19 and positive staining of VIM (Fig. 1b). CCFs showed higher expression of ASMA than SFs (Fig. 1b). The CM from both CCFs revealed a significantly greater ability to induce migration in CCA cells than normal SFA3 and SFA5 cells at 3 and $6 \mathrm{~h}$ (Fig. 1c). CM from CCFs showed no effect on proliferation of CCA cells (Fig. 1d). The expression of CCF-related genes that were previously reported [8], included a disintegrin and matrix metalloproteinase 12 (ADAM12), amphiregulin (AREG), epiregulin $(E R)$, jagged 1 (JAGL1), platelet-derived growth factoralpha (PDGFA), secretogranin 2 (SCG2), periostin $(P N)$, fibroblast activation protein $(F A P)$, fibroblast specific protein (FSP), $A S M A$, and VIM (Additional file 3: Figure S1). $A S M A$ and PDGFA expression levels were higher in CCFs than in SFs (Additional file 3: Figure S1).

\section{Comparing miRNA expression profiles between CCFs and SFs}

The PCR-based miRNA array was performed to measure the miRNA profiling in 2 CCFs compared to 2 SFs. Among 677 human miRNAs, 162 miRNAs were upregulated ( $\geq 2$-fold) and 93 miRNAs were downregulated ( $\leq$ 0.5-fold) in both CCFs (B149 and C096) compared to the expression of either SFA3 or SFA5 cells. The downregulated miRNAs comparing B149 cells to SFs (B149/ SFs) and C096 cells to SFs (C096/SFs) were 17 and 91 miRNAs (Fig. 2a) respectively. Among them, 15 miRNAs (CCFs/SFs) were commonly downregulated in CCFs compared to both SFs after normalization with U6 snRNA (Fig. 2a and Additional file 4: Figure S2). From a literature review, among 15 miRNAs, miR-15a, miR148a and miR-486 targeted several secreted proteins (Additional file 5: Table S3). The expression levels of 
a

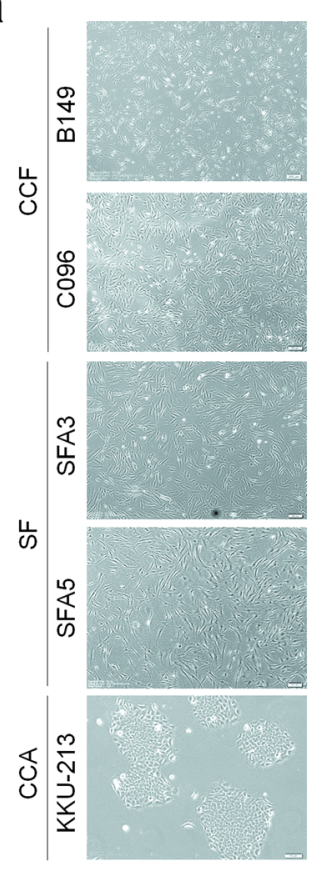

C

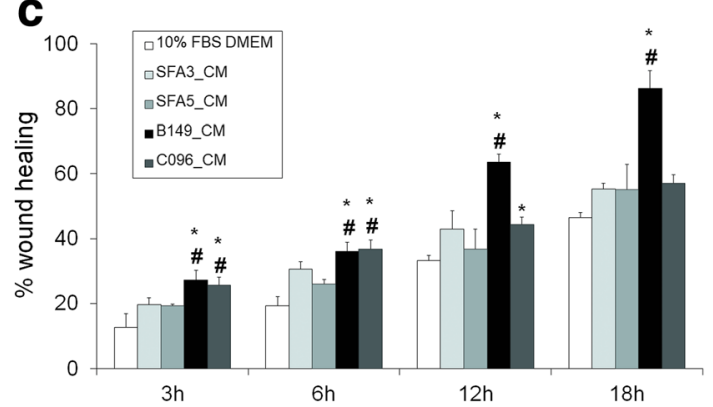

b

U
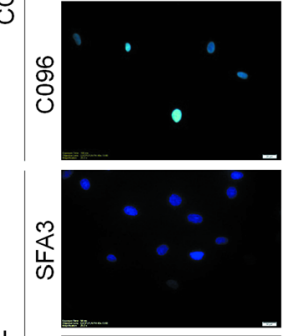

心

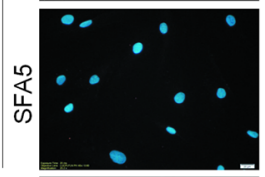

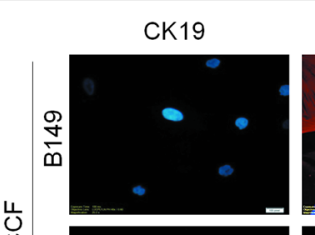
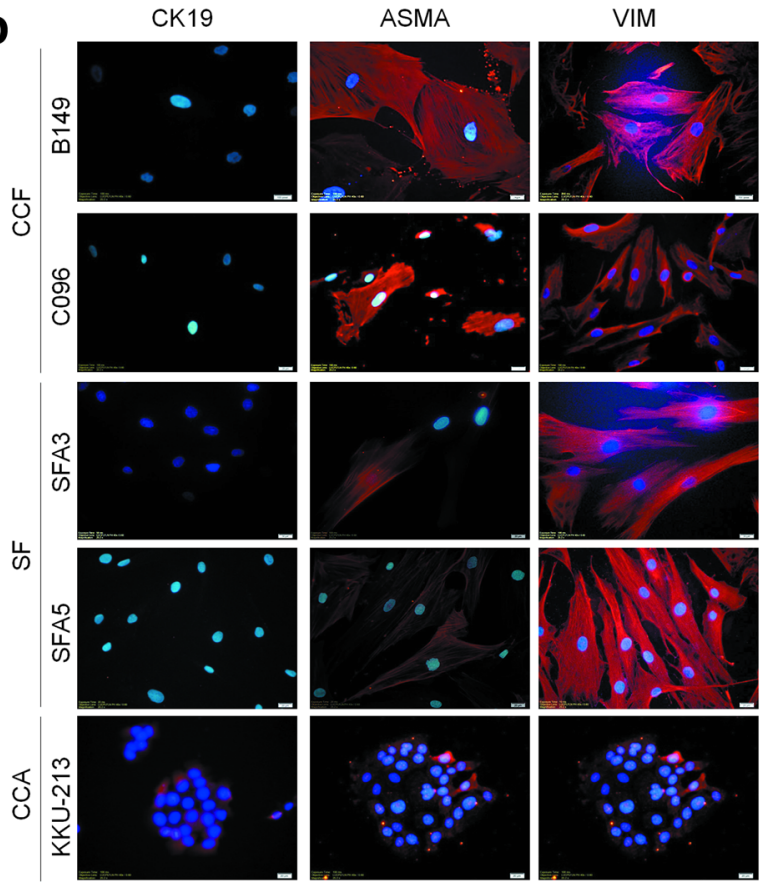

d

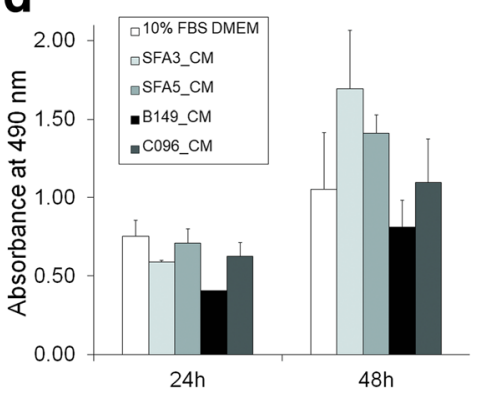

Fig. 1 Characterization of CCFs. a Morphology of CCFs, SFs and CCA cells. Original magnification of 40x. $\mathbf{b}$ Immunocytochemical staining of CK19, VIM and ASMA. The KKU-213 CCA cell line was used as a positive control of CK1 expression. Original magnification of 400x. c The effect of CCFs-CMs and SFs-CMs on migration of CCA cells in a wound healing assay. At 3, 6, 12, and $18 \mathrm{~h}$, the \% of wound area was measured. Bars represent mean \pm SD of three measurements. ${ }^{*} P<0.05$ (compared to 10\% FBS DMEM). ${ }^{*} P<0.05$ (compared to SF_CM). $\mathbf{d}$ The effect of CCFs-CMs and SFs-CMs on proliferation of CCA cells. Bars represent mean \pm SD of two measurements

these miRNAs in CCFs and SFs were examined and only miR-15a and miR-148a showed decreased levels in all CCFs in comparison to those in SFs (Fig. 2b). Moreover, B149/SFs and C096/SFs revealed 145 and 4 up-regulated miRNAs, with 13 miRNAs in common after normalization with U6 snRNA (Additional file 6: Figure S3A and B). In this study, we focused on downregulated miRNAs including miR-15a and miR-148a.

\section{Identification of miR-15a target genes in CCFs}

To predict the potential mRNA targets of miR-15a and miR-148a, TargetScan miRNA target prediction database was used. Taken together, these lists of target genes with the overexpressed genes in CCFs studied by cDNA microarray [8] and highly expressed proteins in the CM (unpublished data), propose several target genes of interest for miR-15a including VEGFA, PAPPA, NRG1, FGF2, PAI-2, AXIN2, FGF7, and WNT3A (Fig. 3a) and for miR-148a are TNFRSF6B, CD62L (L-selectin), TGFA, WNT1, and WNT10B (Additional file 7: Figure S4A). The real-time PCR results revealed that only VEGFA and PAI-2 were up-regulated in CCFs, in comparison with SFs (Fig. 3b). WNT10B was also up-regulated in CCFs, compared with SFs (Additional file 7: Figure S4B).

It has already been shown that WNT10B is a target of miR-148a in CAFs [17]. We confirmed that WNT10B expression was downregulated by ectopic overexpression of miR-148a and was upregulated by the miR-148a inhibitor (Additional file 7: Figure S4C and D). In this study, the focus was on PAI-2 and VEGFA as targets of miR-15a. The expression levels of PAI-2 and VEGFA after transfection of miR-15a mimic in CCFs were 

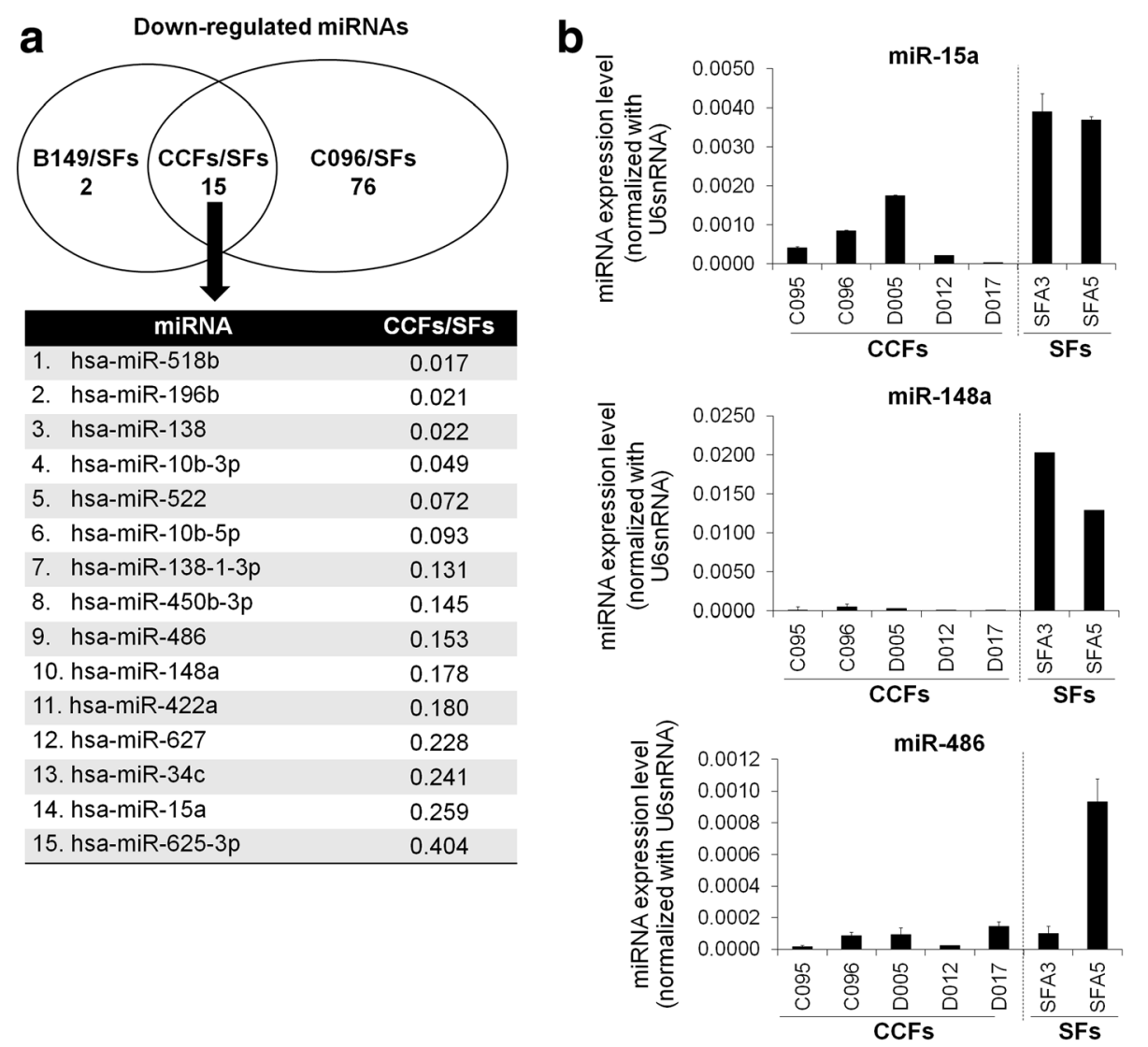

Fig. 2 Comparing miRNA expression profiles between CCFs and SFs. a Vane diagram and lists of down-regulated miRNAs showing the fold change in CCFs versus SFs. $\mathbf{b}$ Real-time PCR of miR-15a, miR-148a and miR-486 expression levels in 5 CCFs and 2 SFs. Bars represent mean \pm SD of three measurements

examined. Reduced expressions of PAI-2 and VEGFA were observed by miR-15a mimic-transfection compared to control miRNA transfection (Fig. 3c and Additional file 8: Figure S5A). Moreover, we confirmed that the miR-15a inhibitor increased the expression of VEGFA and PAI-2 in SFs (Fig. 3d and Additional file 8: Figure S5B).

The mRNA expression levels of these two target genes of miR-15a were checked in CCFs, SFs and CCA cell lines (KKU-213 and KKU-055). Expressions of PAI-2 and VEGFA in CCFs were higher than those in SFs (Fig. 3e and Additional file 8: Figure S5C). Interestingly, expression of PAI-2 in CCF was higher than that in CCA cells (Fig. 3e and f), suggesting that CCFs may be a major source of PAI-2 protein, although cancer cells could also produce PAI-2. In the following experiments, we focused on the miR-15a-PAI-2 axis.

\section{miR-15a-PAI-2 axis in CCFs promotes migration of CCA}

To investigate whether miR-15a can directly target PAI2 by interacting with its $3^{\prime}$-UTR in vitro, we amplified the full-length of 3'-UTR from genomic DNA. The putative miR-15a binding sequences are TGCTGCT. We deleted a deletion mutant of putative miR-15a binding sequences from full-length 3 '-UTR of human PAI-2 construct and used as a negative control. The wild type and mutant 3 '-UTR of PAI- 2 were cloned and inserted downstream of a luciferase reporter gene. Subsequently, miR-15a mimic or control miRNA was co-transfected with these reporter vectors into $293 \mathrm{~T}$ cells. The miR15a mimic significantly decreased the relative luciferase activity of the wild type 3 '-UTR reporter vector, but not statistical significance for the deletion mutant (Fig. 4a).

As PAI-2 is a secreted protein, we confirmed the expression of PAI-2 in CM from C096 cells (Fig. 4b). To examine the effects of PAI- 2 on the proliferation and migration of CCA cells, we used rPAI-2 protein. The rPAI-2 had the capability to increase migration during 6-12 h of exposure compared with untreated controls with a statistical significance in CCA cells (Fig. 4c and d). Notably, rPAI-2 treatment did not promote proliferation (Additional file 9: Figure S6A) or invasion (Additional file 9: Figure S6B, see method in Additional file 10). The level of miR-15a in CCFs was increased after transfection without any cytotoxic effect (data not shown). Next, we examined the effects of CM from 

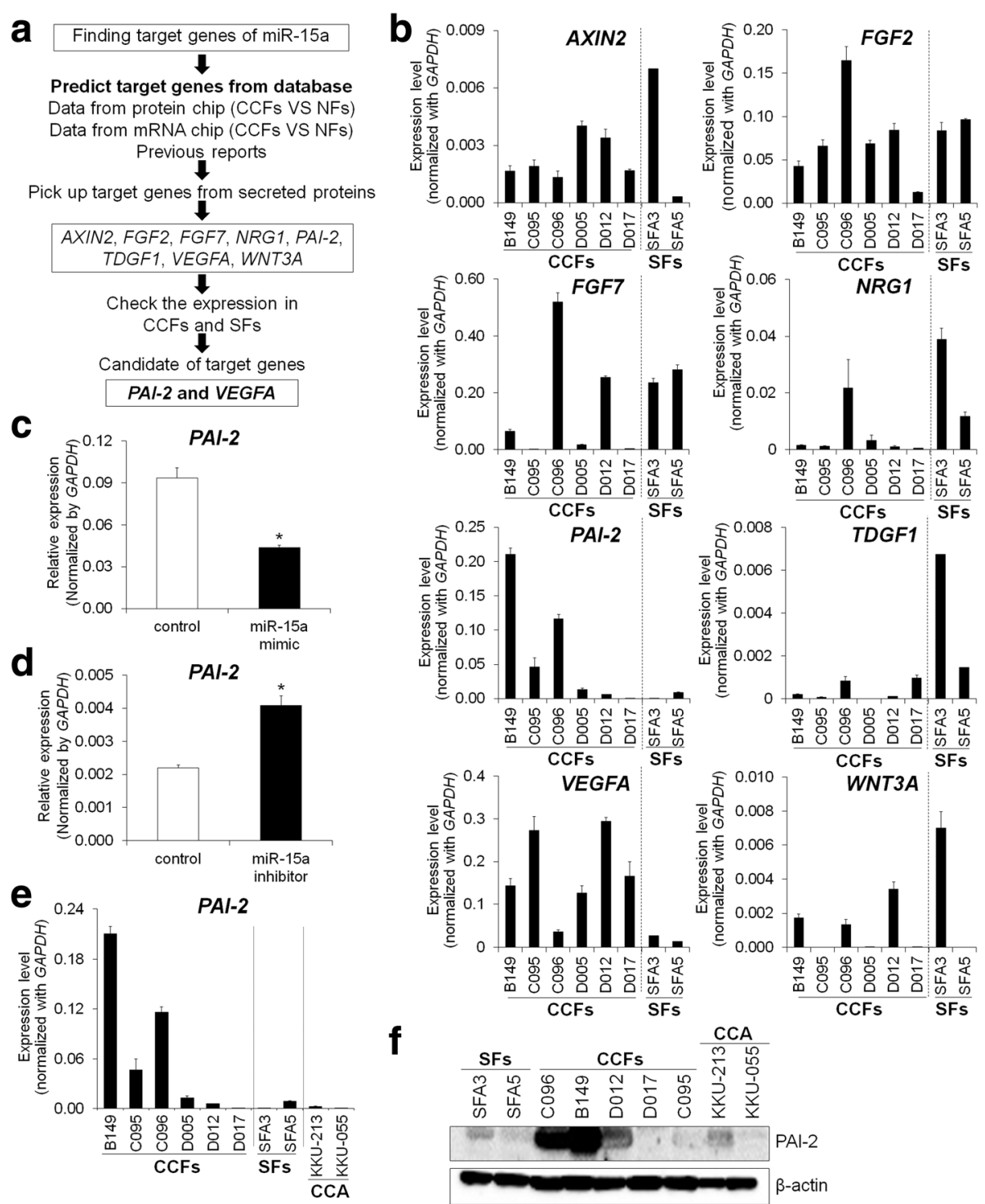

Fig. 3 Identification of miR-15a target genes. a Four criteria for finding the candidate target genes of miR-15a. $\mathbf{b}$ The expression levels of eight predicted target genes of miR-15a in 6 CCFs compared to 2 SFs. Bars represent mean \pm SD of three measurements. c Expression of PAI-2 in miR-15a mimictransfected C096 cells by real-time PCR. Scrambled miRNAs were used as negative control miRNA. $\mathbf{d}$ Expression of PAl-2 in miR-15a inhibitor transfected SFs. ${ }^{*} P<0.05$ compared to control. e Expression of PAl-2 in 6 CCFs, 2 SFs and 2 CCA cell lines. Bars represent means \pm SD of three measurements. $\mathbf{f}$ PAl-2 expression was examined by Western blot analysis in 2 SFs, 5 CCFs, and 2 CCAs. $\beta$-actin was used as a loading control

miR-15a mimic-transfected C096 CCFs on the migration of KKU-055 and KKU-100 CCA cells by the wound healing assay. Ectopic overexpression of miR15a mimic in C096 cells downregulated PAI-2 expression in CM (Fig. 4b). Moreover, the CM from miR-15a mimic-transfected C096 CCFs significantly inhibited the migration of CCA cells in comparison with that from scrambled miRNA transfected cells (Fig. 4e). Interestingly, rPAI-2 treatment partially rescued the inhibitory effect of migration by CM from miR-15a mimic-transfected CCFs in both CCA cell lines (Fig. 4e).
PAI-2 expression in clinical CCA tissues

In 14 CCA and 2 normal liver tissues, expressions of miR-15a and PAI-2 were examined by real-time PCR. Expression of miR-15a in CCA cases was lower than its average expression in normal liver tissues (Fig. 5a). PAI-2 expression was higher in most CCA cases compared with normal liver tissues (Fig. 5b).

Seventy-two clinical samples of CCA cases were enrolled in the immunohistochemistry experiment with the clinicopathological data including sex, age, clinical staging, histological grading, vascular invasion, lymph node 
a

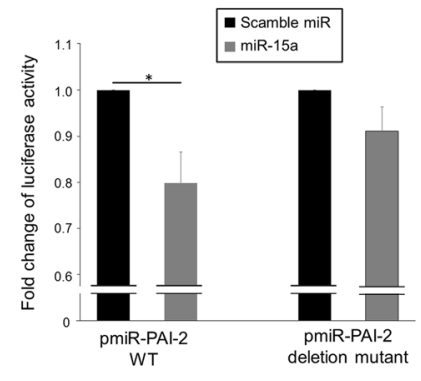

C

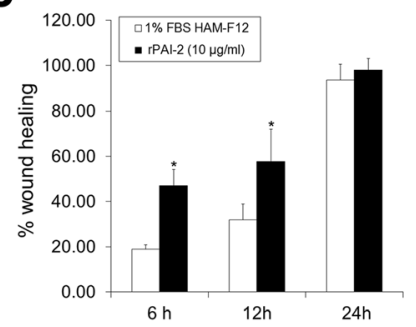

e

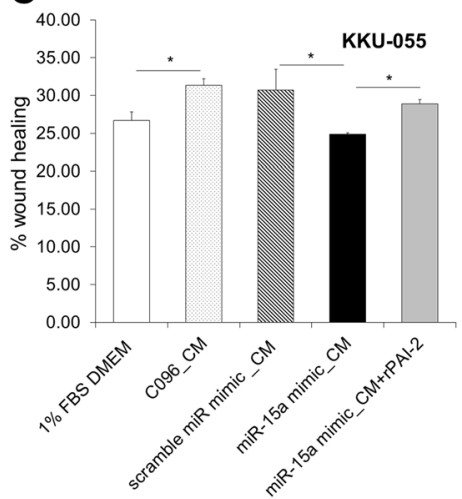

b

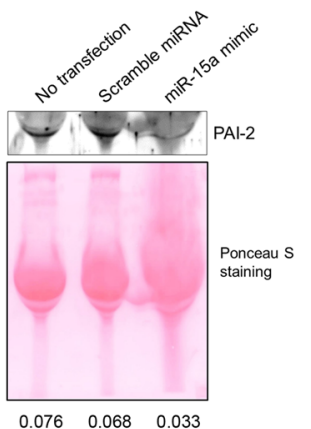

d
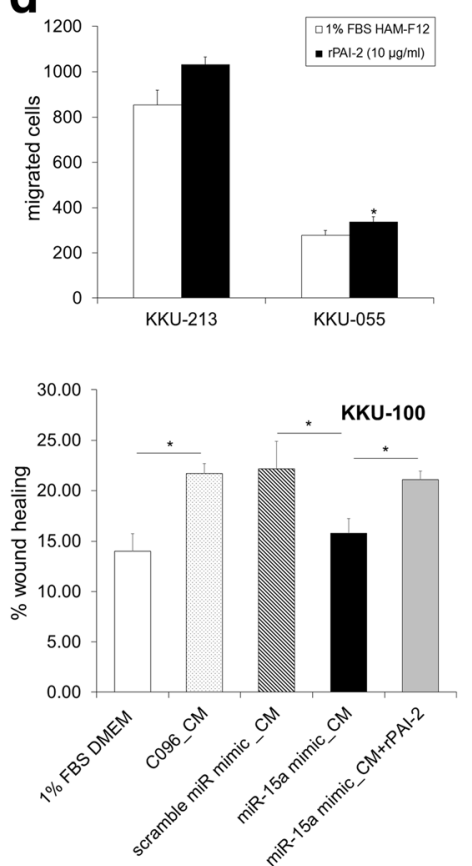

Fig. 4 The miR-15a-PAl-2 axis promotes migration of CCA cells. a Luciferase assay of pmirGLO vector containing wild type 3'-UTR of PAl-2 and its deletion mutant of putative miR-15a binding sequences in mature miR-15a-transfected cells. The data were normalized to scrambled miRNA transfected cells. Bars represent mean \pm SD of five measurements. b Secreted PAl-2 in CM from miR-15a mimic-transfected C096 CCFs examined by Western blot analysis. CMs from cells with no transfection and scrambled miRNA-transfected cells were used as a control. Ponceau $\mathbf{S}$ staining of each sample on the membrane was shown. Densitometric analysis of PAl-2 normalized against the total protein loading is shown. c The effect of rPAl-2 on migration of KKU-213 CCA cells was examined by a wound healing assay at different time points. Graphs show \% of wound areas in KKU-213 cells with or without rPAl-2 in. Bars represent mean \pm SD of three measurements. $\mathbf{d}$ The effect rPAl-2 on CCA cells in the chamber migration assay. Graphs show the number of migrated cells with or without rPAl-2 treatment at $7 \mathrm{~h}$. Bars represent mean \pm SD of three measurements. e The effect of CM from miR-15a mimic or scrambled miRNA transfected C096 CCFs on the migration of CCA cells with or without rPAI-2. The migration was evaluated at $12 \mathrm{~h}$ by a wound healing assay as previously described. Bars represent mean $\pm \mathrm{SD}$ of three measurements. ${ }^{*} P<0.05$

(LN) metastasis and overall survival time (Table 1). The median age of patients was $59 \pm 8.2$ y with a $1.4: 1$ male: female ratio. The average tumor size was $5 \mathrm{~cm}$ for the largest diameter of the mass. Around $75 \%$ of total cases were classified in late stage of the disease (stage IV) and vascular invasion and LN metastasis were found in $33 \%$ of the enrolled cases. The immunostaining revealed positive signals in tumor tissues with different degrees of expression (Fig. 5c). Approximately 63\% of CCA cases (45 of 72) had high PAI-2 levels. Univariate analysis showed that the level of PAI-2 in cancer cells was not correlated with any clinicopathological parameters, but the Cox regression multivariate analysis showed significant correlations of PAI-2 independent of the other parameters (Table 1). The multivariate analysis revealed the potential of a high PAI-2 level being a risk factor in CCA patients at around 3.452 -fold $(P$-value $=0.023$ ). Moreover, high expression of PAI-2 was significantly correlated with histological type and vascular invasion. CCA patients with a high PAI-2 in cancer cells had a median survival time of $249 \mathrm{~d}$ compared with $395 \mathrm{~d}$ in those with low levels. Kaplan-Meier analysis showed a 


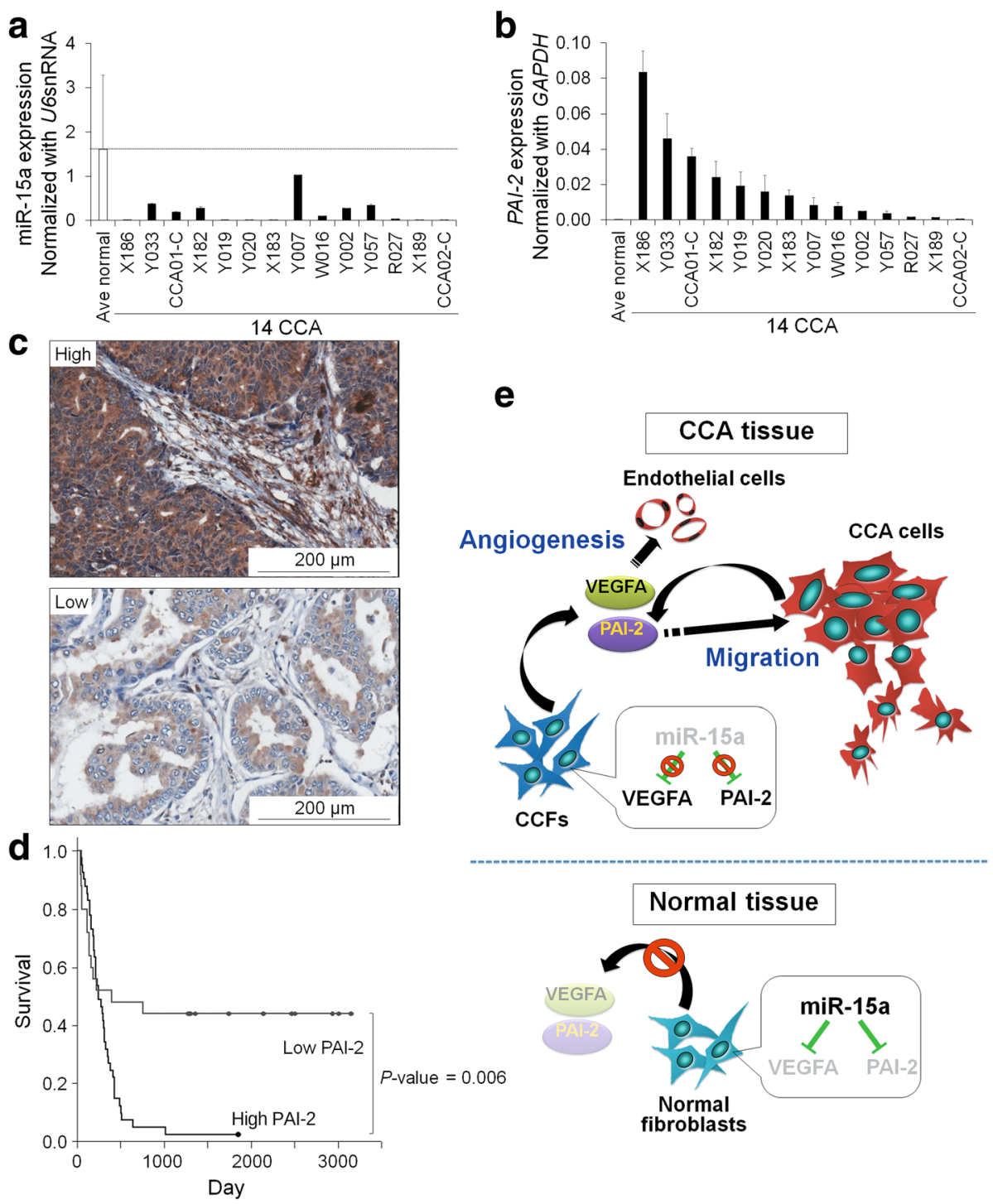

Fig. 5 Expression of PAl-2 in CCA tissues. a Real-time PCR of miR-15a in 14 CCA tissues and 2 normal liver tissues. Bars represent mean \pm SD of three measurements. Dashed line represents the level of average expression in normal liver tissues. $\mathbf{b}$ Real-time PCR of PAl-2 in 14 CCA tissues and 2 normal liver tissues. Bars represent mean \pm SD of three measurements. $\mathbf{c}$ Immunohistochemical staining of PAI- 2 in clinical CCA samples. Representative images of high and low expression of PAI-2 are shown. Scale bar: 200 um. $\mathbf{d}$ The survival analysis by Kaplan-Meier test was performed using 3-y as the cut-off. e The proposed mechanism of miR-15a-PAl-2 axis in microenvironment of CCA tissue

statistically significant correlation of a high PAI-2 level with short survival time (Fig. $5 \mathrm{~d}, P$-value $=0.006$ ).

\section{Discussion}

For decades, CAFs have been recognised for their impact in regulating the aggressive phenotypes of cancer cells in particular through the paracrine activity of secreted substances on cancer cells. In CCA in Thailand, which is commonly caused by the infection of the liver fluke, Opisthorchis viverrini, CAFs have been investigated for their activated phenotype by positive ASMA expression as a poor prognostic marker correlated with short patient survival time [5], recently supported by the finding that aggressive phenotype CAFs in intrahepatic CCA can be identified as a useful predictor for prognosis [18]. Using a mouse model, CCA-bearing animals were targeted with apoptosis induction specifically for CAFs within the tumor masses and the results revealed that CCA growth was attenuated, implicating CAFs in the control of cancer progression [19]. These studies, taken together, provide new evidence that CAFs can serve as potential therapeutic targets in CCA.

Most of the evidence published in the field of CAFs in CCA showed the function of paracrine substances able 
to activate CCA cells $[8,20,21]$. The aberrant production of secreted proteins from CAFs has been identified by us as the results of an alteration in gene expression [8]. Although epigenetic alterations and chromosomal alterations can be found associated with CAFs of different cancer types [22-24], several studies have also shown that alterations of miRNAs in CAFs are common and are involved in multiple functions in tumorigenesis and malignant progression [25-30]. In this study, changes of miRNA expression of CCA CCFs were investigated and miR-15a and miR-148a were selected as the most promising down-regulated miRNAs. The role of miR-15a in CAFs in controlling cancer growth, invasion and resistance to therapy has been identified in prostate cancer [13]. miR-15a, together with miR-16, can target several onco-products i.e. Bcl-2, Bmi-1, Wnt family members, and VEGF, IL-6 [31]. Moreover, VEGFA has been shown to be the target of miR-15a [32,33]. This is the first study to identify PAI-2 as a target of miR-15a. Herein, elevated expression of PAI-2 was observed in CCFs, in comparison with SFs. Moreover, rPAI-2 significantly promoted the migration of CCA cells. Suppression of PAI-2 production from CCFs by transfection of miR-15a mimic attenuated the migration of CCA cells. These findings suggest that the miR-15a-PAI-2 axis in CCFs may be involved in the progression of CCA. Indeed, human CCA tissues with low expression of miR-15a showed high expression of PAI-2. Importantly, patients with high expression of PAI-2 showed poor prognosis.

Moreover, PAI-2 secreted from mast cells can upregulate the expression of ASMA in dermal fibroblasts [34]. This is an important finding which may explain the fibrotic disorder in diseases of excessive fibrosis in the dermis. The precise mechanism by which PAI-2 activates ASMA has not been elucidated. It is proposed that PAI2 may impact cellular activities by changing in the cell surface, including the stability of membrane receptors and extracellular ligands [34]. Hence it is tempting to speculate that PAI-2 secreted into the microenvironment of CCA may activate the expression of ASMA not only in the stromal fibroblasts but also in cancer cells. This may be one of the mechanisms by which PAI-2 can activate CCA cell migration. Another possibility is the finding in urothelial carcinoma that invasive properties were significantly correlated to the up-regulated expression of ASMA in stromal fibroblasts and loss of membranous E-cadherin and increased Snail, Slug, Zeb1 in cancer cells [35]. These altered characters are the hallmark of the 'epithelial-to-mesenchymal' transition phenomenon, increasing cancer cells' ability to migrate and invade. This previous report supports our findings in CCA that ASMA positive stroma (as well as high PAI-2 in tumor microenvironment) correlate with poor prognosis.
In several types of cancer, miRNA expression profiling of CAFs has been reported $[28,36]$. In head and neck cancer, miR-7, miR-196 and miR-335 are significantly up-regulated in CAFs compared with normal fibroblasts, and miR-7 is involved in cancer growth and migration [28]. In prostate cancer, the altered miRNA profile of normal control fibroblasts treated with IL-6 resembled that of CAFs [36]. In breast cancer, 11 dysregulated miRNAs were identified in CAFs: 3 up-regulated miRNAs, miR-221-5p, miR-31-3p, and miR-221-3p, and 8 downregulated miRNAs, miR-205, miR-00b, miR-200c, miR141, miR-101, miR-342-3p, let-7 g, and miR-26b [16]. Here, miR-15a and miR-148a were downregulated in both in vitro CCFs and clinical CCA tissues. This finding is supported by previous reports that miR-148a was downregulated in CAFs from oral cancers compared to normal fibroblasts. Investigation of miR-148a function demonstrated that overexpression of miR-148a in CAFs significantly impaired the migration and invasion of cancer cells by directly targeting WNT10B [17, 37]. In our study, miR-148a was also downregulated and WNT10B was identified as a target gene in CCFs.

The urokinase plasminogen activation (uPA) system is a crucial pathway for tumor invasion and establishment of metastasis. A systematic review evaluating the expression of UPA, urokinase plasminogen activator receptor (uPAR), PAI-1/SerpinE1 and PAI-2/SerpinB2 on primary esophageal, the gastro-esophageal junction, and gastric adenocarcinomas was reported and indicated that a high expression of UPA, UPAR and PAI-1 was associated with a higher risk of disease and poorer prognosis [38]. PAI-1 and PAI-2 are efficient endogenous inhibitors of uPA. They are key players in the breakdown of extracellular matrix and basement membrane [39]. Here, high expression of PAI-2 was observed and correlated with poor prognosis in CCA patients. PAI-2, previously detected in peripheral blood lymphocytes of CCA patients was proposed as part of a set of markers of independent and statistically significant predictors of patient survival [40]. We suggest that in addition to lymphocytes, cancer cells and CCFs may be additional sources of PAI-2 released into the microenvironment of CCA tissues. Importantly, PAI-2 can act in both autocrine and paracrine manners to activate migration of CCA cells.

Base on the association of liver fluke Opisthorchis viverrini infection and CCA oncogenesis, it is interesting to see up-regulated PAI-2 in CCA. PAI- 2 has been reported to be related to the recruitment of M2 macrophages in the nematode infection of the intestine [41]. M2 cells are accepted for a certain time to induce the progressiveness of CCA [42]. Moreover, Schistosoma japonicum granulomas induced mouse liver PAI-2 expression [43]. It is tempting to speculate 
that Opisthorchis viverrini infection may stimulate PAI-2 overproduction released into the CCA microenvironment which may directly induce cancer cell migration (as reported in our study) and at the same time encourage a tumor-promoting environment by modulating infiltrating immune cells. This remains to be tested.

\section{Conclusions}

In conclusion, this is the first report on miRNA profiling in CCFs. Downregulation of miR-15a in CCFs leads to the increased secretion of PAI-2 and VEGFA (Fig. 5e). Our findings provide the opportunity for exploring therapies aimed at reconstituting the miR-15/PAI-2 axis in CCA and the potential of using PAI-2 levels as a poor prognosis marker in these patients.

\section{Additional files}

Additional file 1: Table S1. Primer sequences and product size. (DOCX $16 \mathrm{~kb}$ )

Additional file 2: Table S2. Primer sequences and product size of miRNA-targeted mRNA. (DOCX $17 \mathrm{~kb})$

Additional file 3: Figure S1. The expression of 11 cancer related genes and CAF markers that were previously reported was examined by real-time PCR in CCFs (B149 and C096) and SFs (SFA3 and SFA5). The expression level is normalized to an internal control, GAPDH. Bars represent mean \pm SD of three measurements. (TIFF $180 \mathrm{~kb}$ )

Additional file 4: Figure S2. The expression levels of 15 down-regulated miRNAs in in 2 CCFs (B149 and C096) and 2 normal SFs (SFA3 and SFA5). Expression of miRNAs was examined by real-time PCR. The level is normalized to U6 snRNA. Graph shows the expression of miRNAs in 2 CCFs and the average level of 2 control SFs. (TIFF $194 \mathrm{~kb}$ )

Additional file 5: Table S3. A literature review of 15 miRNAs which are commonly down-regulated in CCFs. (PPTX $53 \mathrm{~kb}$ )

Additional file 6: Figure S3. The up-regulated miRNAs in CCFs. (A) Vane diagram and lists of 13 up-regulated miRNAs showing the folding compared to that in SFs. (B) Expression of up-regulated miRNAs in 2 CCFs (B149 and C096) and 2 normal SFs (SFA3 and SFA5) was examined by real-time PCR. Graph shows the expression of miRNAs in 2 CCFs and the average level of 2 control SFs. (TIFF $212 \mathrm{~kb}$ )

Additional file 7: Figure S4. List of candidate target genes of miR148a. (A) Four criteria of finding the candidate target genes of miR-148a. (B) The expression levels of five predicted target genes of miR-148a including WNT10B, TGFA, WNT1, TNFRSF6B and L-selectin in CCFs and SFs. Scrambled miRNAs were used as a negative control. Bars represent mean \pm SD of three measurements in one experiment. (C) Expression of WNT10B in miR-148a mimic transfected C096 CCFs was examined by real-time PCR. Bars represent mean \pm SD of three measurements. ${ }^{*} P \leq 0.05$ compared to control. (D) Expression of WNT1OB in miR-148a inhibitor transfected SFA3 SFs was examined by real-time PCR. Bars represent mean \pm SD of three measurements. ${ }^{*} P \leq 0.05$ compared to control. (TIFF $200 \mathrm{~kb}$ )

Additional file 8: Figure S5. $V E G F A$ is a target gene of miR-15a. (A) Expression of VEGFA in miR-15a mimic transfected C096 CCFs was examined by real-time PCR. Scrambled miRNAs were used as negative control miRNA. Bars represent mean \pm SD of three measurements. (B) Expression of VEGFA in miR-15a inhibitor transfected SFA3 SFs was examined by real-time PCR. Bars represent mean \pm SD of three measurements. (C) Expression of VEGFA in 6 CCFs (B149, C095, C096, D005, D012, and D017), SFs (SFA3 and SFA5) and CCA cell lines (KKU-213 and KKU-055) was examined by real-time $P C R$. Bars represent means \pm SD of three measurements. ${ }^{*} P \leq 0.05$ compared to control. (TIFF $78 \mathrm{~kb}$ )
Additional file 9: Figure S6. The effects of rPAI-2 on CCA tumorigenic properties. (A) Cell proliferation was examined by WST assay in KKU-213 and KKU-055 at 24, 48, and $72 \mathrm{~h}$ after $10 \mu \mathrm{g} / \mathrm{ml}$ of rPAl-2 treatment. The $2 \%$ FBS containing media are used as negative controls. (B) Cell invasion by Transwell ${ }^{\oplus}$ invasion assay in KKU-213 and KKU-055. After incubation for $18 \mathrm{~h}$ with $10 \mu \mathrm{g} / \mathrm{ml}$ of rPAl-2, invaded cells were counted. Bars represent mean $\pm \mathrm{SD}$ of three measurements. ${ }^{*} P<0.05$ compared to control. (TIFF $4367 \mathrm{~kb}$ )

Additional file 10: Supplementary information of the invasion assay method. (DOCX $12 \mathrm{~kb}$ )

\section{Abbreviations}

ASMA: Alpha smooth muscle actin; CAFs: Cancer-associated fibroblasts; CCA: Cholangiocarcinoma; CCFs: CCA-associated fibroblasts; miR: MicroRNA; PAl-2: Plasminogen activator inhibitor-2; SFs: Skin fibroblasts

\section{Acknowledgements}

The authors would like to thanks Prof. James A. Will, University of Wisconsin, Madison, USA and Prof. Suzanne A. Eccles for English editing. Special thanks to Ms. Nawarat Boonmee for the immunohistochemistry techniques.

\section{Funding}

This work has been supported by Royal Golden Jubilee (RGJ), Thailand Research Fund (TRF) to PU (Grant no. PHD/0299/2550). The research grant from TRF (Grant no. RSA5480003) was given to CT. This research project is partly supported by Faculty of Medicine Siriraj Hospital, Mahidol University.

Availability of data and materials

The datasets obtained and/or analyzed during the current study are available from the corresponding author in reasonable request.

\section{Authors' contributions}

PU performed experiments and summarized the results. KV, SW, CP and PK prepared human cancer tissues from the Khon Kaen site and collected clinicopathological data. T, PJ and NI performed some experiments. YS and AP prepared human cancer tissues from the Mahidol site. PT helped design the experiments and data analysis. CT and YK designed the study, analyzed the results and wrote the manuscript. All authors read and approved the final manuscript.

\section{Ethics approval and consent to participate}

The human cancer tissues used in this study were approved by the institute ethical committee of Mahidol University and Khon Kaen University.

\section{Consent for publication}

Not applicable.

\section{Competing interests}

The authors declare that they have no competing interests.

\section{Publisher's Note}

Springer Nature remains neutral with regard to jurisdictional claims in published maps and institutional affiliations.

\section{Author details}

${ }^{1}$ Graduate Program in Immunology, Department of Immunology, Faculty of Medicine Siriraj Hospital, Mahidol University, Bangkok, Thailand. ²Department of Biochemistry, Faculty of Medicine, Khon Kaen University, Khon Kaen, Thailand. ${ }^{3}$ Department of Pathology, Faculty of Medicine, Khon Kaen University, Khon Kaen, Thailand. ${ }^{4}$ Department of Surgery, Faculty of Medicine, Khon Kaen University, Khon Kaen, Thailand. ${ }^{5}$ Cholangiocarcinoma Research Institute, Khon Kaen University, Khon Kaen, Thailand. ${ }^{6}$ Department of Oral Molecular Pathology, Tokushima University Graduate School of Biomedical Sciences, Tokushima, Japan. ${ }^{7}$ Department of Surgery, Faculty of Medicine Siriraj Hospital, Mahidol University, Bangkok, Thailand. ${ }^{8}$ Department of Pathology, Faculty of Medicine Siriraj Hospital, Mahidol University, Bangkok, Thailand. 'Department of Immunology, Faculty of Medicine Siriraj Hospital, Mahidol University, Bangkok, Thailand. 


\section{Received: 10 September 2017 Accepted: 4 January 2018}

\section{Published online: 18 January 2018}

\section{References}

1. Gatto M, Bragazzi MC, Semeraro R, Napoli C, Gentile R, Torrice A, Gaudio E, Alvaro D. Cholangiocarcinoma: update and future perspectives. Dig Liver Dis. 2010;42(4):253-60

2. Sripa B, Bethony JM, Sithithaworn P, Kaewkes S, Mairiang E, Loukas A, Mulvenna J, Laha T, Hotez PJ, Brindley PJ. Opisthorchiasis and Opisthorchisassociated cholangiocarcinoma in Thailand and Laos. Acta Trop. 2011; 120(Suppl 1):S158-68.

3. Sirica AE, Dumur Cl, Campbell DJ, Almenara JA, Ogunwobi OO, Dewitt JL. Intrahepatic cholangiocarcinoma progression: prognostic factors and basic mechanisms. Clin Gastroenterol Hepatol. 2009;7(11 Suppl):S68-78.

4. Terada T, Makimoto K, Terayama N, Suzuki Y, Nakanuma Y. Alpha-smooth muscle actin-positive stromal cells in cholangiocarcinomas, hepatocellular carcinomas and metastatic liver carcinomas. J Hepatol. 1996;24(6):706-12.

5. Chuaysri $C$, Thuwajit P, Paupairoj A, Chau-In S, Suthiphongchai T, Thuwajit $C$. Alpha-smooth muscle actin-positive fibroblasts promote biliary cell proliferation and correlate with poor survival in cholangiocarcinoma. Oncol Rep. 2009;21(4):957-69.

6. Cirri $P$, Chiarugi P. Cancer-associated-fibroblasts and tumour cells: a diabolic liaison driving cancer progression. Cancer Metastasis Rev. 2012;31(1-2):195-208.

7. Pietras K, Ostman A. Hallmarks of cancer: interactions with the tumor stroma. Exp Cell Res. 2010;316(8):1324-31.

8. Utispan K, Thuwajit P, Abiko Y, Charngkaew K, Paupairoj A, Chau-in S, Thuwajit C. Gene expression profiling of cholangiocarcinoma-derived fibroblast reveals alterations related to tumor progression and indicates periostin as a poor prognostic marker. Mol Cancer. 2010;9:13.

9. Bartel DP. MicroRNAs: genomics, biogenesis, mechanism, and function. Cell. 2004;116(2):281-97.

10. Esquela-Kerscher A, Slack FJ. Oncomirs - microRNAs with a role in cancer. Nat Rev Cancer. 2006;6(4):259-69.

11. Bronisz A, Godlewski J, Wallace JA, Merchant AS, Nowicki MO, Mathsyaraja $H$, Srinivasan R, Trimboli AJ, Martin CK, Li F, et al. Reprogramming of the tumour microenvironment by stromal PTEN-regulated miR-320. Nat Cell Biol. 2011;14(2):159-67.

12. Enkelmann A, Heinzelmann J, von Eggeling F, Walter M, Berndt A, Wunderlich $\mathrm{H}$, Junker K. Specific protein and miRNA patterns characterise tumour-associated fibroblasts in bladder cancer. J Cancer Res Clin Oncol. 2011;137(5):751-9.

13. Musumeci M, Coppola V, Addario A, Patrizii M, Maugeri-Sacca M, Memeo L, Colarossi C, Francescangeli F, Biffoni M, Collura D, et al. Control of tumor and microenvironment cross-talk by miR-15a and miR-16 in prostate cancer. Oncogene. 2011;30(41):4231-42.

14. Nielsen BS, Jorgensen S, Fog JU, Sokilde R, Christensen IJ, Hansen U, Brunner N, Baker A, Moller S, Nielsen HJ. High levels of microRNA-21 in the stroma of colorectal cancers predict short disease-free survival in stage II colon cancer patients. Clin Exp Metastasis. 2011;28(1):27-38.

15. Rask L, Balslev E, Jorgensen S, Eriksen J, Flyger H, Moller S, Hogdall E, Litman T, Nielsen BS. High expression of miR-21 in tumor stroma correlates with increased cancer cell proliferation in human breast cancer. APMIS. 2011; 119(10):663-73.

16. Zhao L, Sun Y, Hou Y, Peng Q, Wang L, Luo H, Tang X, Zeng Z, Liu M. MiRNA expression analysis of cancer-associated fibroblasts and normal fibroblasts in breast cancer. Int J Biochem Cell Biol. 2012;44(11):2051-9.

17. Aprelikova O, Palla J, Hibler B, Yu X, Greer YE, Yi M, Stephens R, Maxwell GL, Jazaeri A, Risinger $\mathrm{J}$, et al. Silencing of miR-148a in cancer-associated fibroblasts results in WNT10B-mediated stimulation of tumor cell motility. Oncogene. 2013;32(27):3246-53.

18. Zhang XF, Dong M, Pan $Y H$, Chen JN, Huang XQ, Jin $Y$, Shao CK: Expression pattern of cancer-associated fibroblast and its clinical relevance in intrahepatic cholangiocarcinoma. Hum Pathol. 2017; pii: S00468177(17)30132-6. doi: https://doi.org/10.1016/j.humpath.2017.04.014.

19. Mertens JC, Fingas CD, Christensen JD, Smoot RL, Bronk SF, Werneburg NW Gustafson MP, Dietz AB, Roberts LR, Sirica AE, et al. Therapeutic effects of deleting cancer-associated fibroblasts in cholangiocarcinoma. Cancer Res. 2013;73(2):897-907.

20. Sirica AE, Almenara JA, Li C. Periostin in intrahepatic cholangiocarcinoma: pathobiological insights and clinical implications. Exp Mol Pathol. 2014; 97(3):515-24.
21. Sirica AE, Campbell DJ, Dumur Cl. Cancer-associated fibroblasts in intrahepatic cholangiocarcinoma. Curr Opin Gastroenterol. 2011;27(3): 276-84.

22. Ishiguro K, Yoshida T, Yagishita H, Numata Y, Okayasu T. Epithelial and stromal genetic instability contributes to genesis of colorectal adenomas. Gut. 2006;55(5):695-702.

23. Tuhkanen H, Anttila M, Kosma VM, Heinonen S, Juhola M, Helisalmi S, Kataja $V$, Mannermaa A. Frequent gene dosage alterations in stromal cells of epithelial ovarian carcinomas. Int J Cancer. 2006;119(6):1345-53.

24. Zhang MW, Fujiwara K, Che X, Zheng S, Zheng L. DNA methylation in the tumor microenvironment. J Zhejiang Univ Sci B. 2017;18(5):365-72.

25. Li J, Guan J, Long X, Wang Y, Xiang X. Mir-1-mediated paracrine effect of cancer-associated fibroblasts on lung cancer cell proliferation and chemoresistance. Oncol Rep. 2016;35(6):3523-31.

26. Li X, Fan Q, Li J, Song J, Gu Y. MiR-124 down-regulation is critical for cancer associated fibroblasts-enhanced tumor growth of oral carcinoma. Exp Cell Res. 2017;351(1):100-8.

27. Schoepp M, Strose AJ, Haier J: Dysregulation of miRNA expression in cancer associated fibroblasts (CAFs) and its consequences on the tumor microenvironment. Cancers (Basel). 2017; 9(6). May 24; pii: E54: doi: https:// doi.org/10.3390/cancers9060054.

28. Shen Z, Qin X, Yan M, Li R, Chen G, Zhang J, Chen W. Cancer-associated fibroblasts promote cancer cell growth through a miR-7-RASSF2-PAR-4 axis in the tumor microenvironment. Oncotarget. 2017:8(1):1290-303.

29. Wang Z, Tan Y, Yu W, Zheng S, Zhang S, Sun L, Ding K. Small role with big impact: miRNAs as communicators in the cross-talk between cancer-associated fibroblasts and cancer cells. Int J Biol Sci. 2017;13(3): 339-48.

30. Yan Y, Wang R, Guan W, Qiao M, Wang L. Roles of microRNAs in cancer associated fibroblasts of gastric cancer. Pathol Res Pract. 2017;213(7):730-6.

31. Karaa ZS, lacovoni JS, Bastide A, Lacazette E, Touriol C, Prats H. The VEGF IRESes are differentially susceptible to translation inhibition by miR-16. RNA. 2009;15(2):249-54

32. Liu XJ, Bai $X G$, Teng $Y L$, Song $L$, Lu N, Yang RQ. miRNA-15a-5p regulates VEGFA in endometrial mesenchymal stem cells and contributes to the pathogenesis of endometriosis. Eur Rev Med Pharmacol Sci. 2016;20(16): 3319-26.

33. Chen $\mathrm{H}$, Tian Y. MiR-15a-5p regulates viability and matrix degradation of human osteoarthritis chondrocytes via targeting VEGFA. Biosci Trends. 2017; 10(6):482-8.

34. Kim CD, Sohn KC, Lee SS, Lee JH, Kim S, Lee YH, Ryu EK, Park YS. Plasminogen activator inhibitor-2 (PAI-2) secreted from activated mast cells induces alpha-smooth muscle actin (alpha-SMA) expression in dermal fibroblasts. J Dermatol Sci. 2011;62(3):204-6.

35. Schulte J, Weidig M, Balzer P, Richter P, Franz M, Junker K, Gajda M, Friedrich $\mathrm{K}$, Wunderlich $\mathrm{H}$, Ostman $\mathrm{A}$, et al. Expression of the E-cadherin repressors snail, slug and Zeb1 in urothelial carcinoma of the urinary bladder: relation to stromal fibroblast activation and invasive behaviour of carcinoma cells. Histochem Cell Biol. 2012;138(6):847-60.

36. Doldi V, Callari M, Giannoni E, D'Aiuto F, Maffezzini M, Valdagni R, Chiarugi P, Gandellini P, Zaffaroni N. Integrated gene and miRNA expression analysis of prostate cancer associated fibroblasts supports a prominent role for interleukin-6 in fibroblast activation. Oncotarget. 2015:6(31):31441-60.

37. Min A, Zhu C, Peng S, Shuai C, Sun L, Han Y, Qian Y, Gao S, Su T. Downregulation of Microrna-148a in cancer-associated fibroblasts from oral cancer promotes cancer cell migration and invasion by targeting Wnt10b. J Biochem Mol Toxicol. 2016;30(4):186-91.

38. Brungs D, Chen J, Aghmesheh M, Vine KL, Becker TM, Carolan MG, Ranson M. The urokinase plasminogen activation system in gastroesophageal cancer: a systematic review and meta-analysis. Oncotarget. 2017:8(14):23099-109.

39. Su SC, Lin CW, Yang WE, Fan WL, Yang SF. The urokinase-type plasminogen activator (UPA) system as a biomarker and therapeutic target in human malignancies. Expert Opin Ther Targets. 2016;20(5):551-66.

40. Subimerb C, Wongkham C, Khuntikeo N, Leelayuwat C, McGrath MS, Wongkham S. Transcriptional profiles of peripheral blood leukocytes identify patients with cholangiocarcinoma and predict outcome. Asian Pac J Cancer Prev. 2014;15(10):4217-24.

41. Zhao A, Yang Z, Sun R, Grinchuk V, Netzel-Arnett S, Anglin IE, Driesbaugh $\mathrm{KH}$, Notari L, Bohl JA, Madden KB, et al. SerpinB2 is critical to Th2 immunity against enteric nematode infection. J Immunol. 2013;190(11):5779-87. 
42. Thanee M, Loilome W, Techasen A, Namwat N, Boonmars T, Pairojkul C, Yongvanit P. Quantitative changes in tumor-associated M2 macrophages characterize cholangiocarcinoma and their association with metastasis. Asian Pac J Cancer Prev. 2015;16(7):3043-50.

43. Schroder WA, Gardner J, Le TT, Duke M, Burke ML, Jones MK, McManus DP, Suhrbier A. SerpinB2 deficiency modulates Th1Th2 responses after schistosome infection. Parasite Immunol. 2010;32(11-12):764-8.

Submit your next manuscript to BioMed Central and we will help you at every step:

- We accept pre-submission inquiries

- Our selector tool helps you to find the most relevant journal

- We provide round the clock customer support

- Convenient online submission

- Thorough peer review

- Inclusion in PubMed and all major indexing services

- Maximum visibility for your research

Submit your manuscript at www.biomedcentral.com/submit
Biomed Central 